\title{
A COMPARATIVE CLINICAL STUDY ON THE EFFICACY OF HINGWADI GRUTHAM AS NASYA, PANA AND BOTH IN THE MANAGEMENT OF KHAPHAJA UNMADA WITH SPECIAL REFERENCE TO DEPRESSIVE DISORDERS
}

\author{
*Dr. Devulapally Anuradha ${ }^{1,2}$ \\ ${ }^{1}$ Asst.Professor and HOD, Dept. of Agadatantra, Dr. BRKR, G.A.C, Hyd. A.P., INDIA \\ ${ }^{2} \mathrm{PhD}$ Scholar, Dr. NTR University of Health Sciences, Vijayawada, A.P, INDIA \\ *Corresponding author's Email: dranud@yahoo.com
}

Received 24 August 2012; Review Completed 04 Sep 2012; Accepted 08 Sep 2012, Available online 15 Sep 2012

\begin{abstract}
:
Mental disorders are well known for long causing a variety of psychiatric diseases. Ayurveda the majestic legacy of our rich Indian heritage has provided an ocean of knowledge regarding mind and its management. Though a number of formulations are there in classics of Ayurveda, the specific medicine which acts effectively on kaphaja unmada in correlation with depression is explored here ,because treating depression is the need of the hour. Depression is converting majority of people into mentally weak and in-active personalities with incidental tendencies, which in turn is harming the whole humanity. This study aims at establishing the efficacy of Hingwadi grutha in decreasing the score of Hamilton's Rating Scale for depression and kaphaja unmada rating scale. It is an experimental study with pre-test and post- test designs. All the 30 cases of kaphaja unmades were selected randomly from the O.P.D of APPRC-Hyd. The design in this research consists of three groups A, B and C. In group 'A' 10 Patients were given only Hingwadi grutha nasyam (nasal drops) with 8 drops twice a day for 45 days. In group 'B' 10 patients were given Hingwadi grutha panam (internally) $10 \mathrm{ml}$ twice daily for 45 days. In group ' $\mathrm{C}$ ' 10 patients were given both the treatments; Nasal drops (Nasya) and oral administration (Pana) of Hingwadi grutha for 45 days. In group 'A' relief was 42.93\% ( $\mathrm{P}<0.0001)$. The change was significant. In group ' $\mathrm{B}$ ' the relief was $47.09 \%(\mathrm{P}<0.0001)$. The change was significant. There was a relief of $76.35 \%(\mathrm{P}<0.0001)$ in the group $\mathrm{C}$ which is highly significant. The change that occurred in the three groups weres statistically significant. Kaphaja unmada rating scale results were found highly significant $(\mathrm{P}<0.0001)$ in group ' $\mathrm{C}$ ' when compared to group ' $\mathrm{A}$ ' and group ' $\mathrm{B}$ '. Hamilton depression rating scale results were also found to be highly significant $(\mathrm{P}<0.0001)$ in group ' $C$ ' when compared with group 'A' \& 'B'. The symptoms of kaphaja unmada and depression are approximately same. Female population is more prone to the ailment. Positive family history, stress and unsatisfied life also contribute for the ailment. The sample size was small. No objective parameters were used. The short period of study limited long term observations.

Key Words: Unmada, Depressive disorders, Hingwadigrutha, Nasyakarma \& Pana karma.
\end{abstract}

\section{INTRODUCTION:}

'Unmada' is the common term for mental abnormalities in Ayurveda ${ }^{1 .}$ The exact correlation of Ayurvedic mental disorders to Modern is not easy because of differences in diagnostic criteria, psychopathologies, basic theories (biomedical theories in modern medicine \& Dosha theory in Ayurveda) etc. But if we look into the symptoms of kaphaonmada and symptoms of depressive disorders we can find that more that $70 \%$ of the symptoms are same ${ }^{2}$. The disease entity is since Vedic period ${ }^{3}$. In the present age of astonishing scientific advancements in almost every aspect of human life, one faculty man is still unable to gain authority on is Human mind. Mismanagement of mind is causing a variety of Psychiatric diseases. Modern lifestyle forces people to ignore the harmful changes in their mind which gradually affects their body, leaving them fit for nothing. 4 .

Depression is a major psychiatric ailment affecting $9.5 \%$ of world population 5. It affects the body moods and thoughts. Ayurveda provides rational means for the treatment of many physical \& mental disorders that are considered incurable in other systems of medicine. As a line of therapy to treat at gross root level, nasya karma \& pana karma with Hingwadigrutha were selected ${ }^{6}$. In unmada chikista grutha alone is also effective. But if it is given along with herbal preparations the results are utmost

\section{MATERIALS AND METHODS:}

30 patients were randomly selected who were suffering from kaphaja unmada \& depressive disorders from APPRC-Hyd. and were divided into 3 groups of 10 patients each. In group ' $A$ ' 10 patients were treated only with Hingwadi grutha nasyam (nasal drops.) 4 drops in each nostril twice a day for 45 days.

In group 'B' 10 patients were treated with Hingwadi grutha panam (orally) $10 \mathrm{ml}$ with luke warm milk twice daily.

In group ' $\mathrm{C}$ ' 10 patients were treated with both nasya \& pana (Nasal drops \& oral oil respectively) given for 45 days. Before starting the treatment all the three groups of patients have undergone vamanakarma at GAH-Erragadda.

\section{Inclusion Criteria:}

1) Patients with kaphaja unmada rating score

2) Age group 15-50yrs irrespective of sex, race ,caste \& religion.

\section{Exclusion Criteria:}

1) Other types of Nija \& Aganthuja (Hereditary or Accidental) respectively. 
Anuradha et al

Journal of Drug Delivery \& Therapeutics; 2012, 2(5), 90-96

2) Presence of their organic and neurological disorders.

\section{Parameters:}

The signs and symptoms of kaphaja unmada described in the Ayurvedic classics were taken to prepare kaphaja unmada rating scale. It was used along with Hamilton's rating scale of depression as the tool for assessment of the variables.

\section{OBSERVATIONS \& RESULTS:}

Table 1: Distribution of 30 patients of Kaphaja unmada according to Sex

\begin{tabular}{|l|l|l|l|l|l|l|l|}
\hline Sl.No & \multirow{2}{*}{ Sex } & \multicolumn{2}{|l|}{ Group 'A' } & Group 'B' & \multicolumn{2}{l|}{ Group 'C' } \\
\cline { 3 - 8 } & $\begin{array}{l}\text { No. of } \\
\text { Patients }\end{array}$ & Percentage & $\begin{array}{l}\text { No. of } \\
\text { Patients }\end{array}$ & Percentage & $\begin{array}{l}\text { No. } \\
\text { Patients }\end{array}$ & of \\
\hline 1 & Male & 3 & $30 \%$ & 6 & $60 \%$ & 4 & $40 \%$ \\
\hline 2 & Female & 7 & $70 \%$ & 4 & $40 \%$ & 6 & $60 \%$ \\
\hline Total & 10 & $100 \%$ & 10 & $100 \%$ & 10 & $100 \%$ \\
\hline
\end{tabular}

Table 2: Distribution of 30 patients of Kaphaja unmada (Depression) according to Age

\begin{tabular}{|l|l|l|l|l|l|l|l|}
\hline Sl.No & \multirow{2}{*}{ Age } & \multicolumn{2}{|l|}{ Group 'A' } & \multicolumn{2}{l|}{ Group 'B' } & \multicolumn{2}{l|}{ Group 'C' } \\
\cline { 3 - 8 } & $\begin{array}{l}\text { No. of } \\
\text { Patients }\end{array}$ & Percentage & $\begin{array}{l}\text { No. of } \\
\text { Patients }\end{array}$ & Percentage & $\begin{array}{l}\text { No. of of } \\
\text { Patients }\end{array}$ & Percentage \\
\hline 1 & $15-25$ & 1 & $10 \%$ & 2 & $20 \%$ & 3 & $30 \%$ \\
\hline 2 & $25-35$ & 3 & $30 \%$ & 4 & $40 \%$ & 2 & $20 \%$ \\
\hline 3 & $35-45$ & 4 & $40 \%$ & 2 & $20 \%$ & 4 & $40 \%$ \\
\hline 4 & $45-55$ & 2 & $20 \%$ & 2 & $20 \%$ & 1 & $10 \%$ \\
\hline Total & 10 & $100 \%$ & 10 & $100 \%$ & 10 & $100 \%$ \\
\hline
\end{tabular}

Table 3: Distribution of 30 patients of Kaphaja unmada (Depression) according to Education

\begin{tabular}{|l|l|l|l|l|l|l|l|}
\hline Sl.No & Education & \multicolumn{3}{|l|}{ Group 'A' } & \multicolumn{2}{l|}{ Group 'B' } & \multicolumn{2}{l|}{ Group 'C' } \\
\cline { 3 - 8 } & & $\begin{array}{l}\text { No. of } \\
\text { Patients }\end{array}$ & Percentage & $\begin{array}{l}\text { No. of } \\
\text { Patients }\end{array}$ & Percentage & $\begin{array}{l}\text { No. of of } \\
\text { Patients }\end{array}$ & Percentage \\
\hline 1 & Un educated & - & $0 \%$ & 1 & $10 \%$ & - & - \\
\hline 2 & Primary & 2 & $20 \%$ & 1 & $10 \%$ & 3 & $30 \%$ \\
\hline 3 & Secondary & 1 & $10 \%$ & 5 & $50 \%$ & 5 & $50 \%$ \\
\hline 4 & $\begin{array}{l}\text { Higher } \\
\text { Secondary }\end{array}$ & 3 & $30 \%$ & 3 & $30 \%$ & 1 & $10 \%$ \\
\hline 5 & Graduate & 4 & $40 \%$ & 1 & $10 \%$ & 1 & $10 \%$ \\
\hline
\end{tabular}

Table 4: Distribution of 30 patients of Kaphaja unmada according to socio-economic status

\begin{tabular}{|l|l|l|l|l|l|l|l|}
\hline Sl.No & $\begin{array}{l}\text { Socio- } \\
\text { Economic }\end{array}$ & \multicolumn{2}{|l|}{ Group 'A' } & \multicolumn{2}{l|}{ Group 'B' } & \multicolumn{2}{l|}{ Group 'C' } \\
\cline { 2 - 7 } & $\begin{array}{l}\text { No. of } \\
\text { Patients }\end{array}$ & Percentage & $\begin{array}{l}\text { No. of } \\
\text { Patients }\end{array}$ & Percentage & $\begin{array}{l}\text { No. } \\
\text { Patients }\end{array}$ & of \\
\hline 1 & Poor & - & - & 4 & $40 \%$ & 2 & $20 \%$ \\
\hline 2 & Middle & 10 & $100 \%$ & 6 & $60 \%$ & 8 & $80 \%$ \\
\hline Total & & 10 & $100 \%$ & 10 & $100 \%$ & 10 & $100 \%$ \\
\hline
\end{tabular}

Table 5: Distribution of 30 patients of Kaphaja unmada according to marital status

\begin{tabular}{|l|l|l|l|l|l|l|l|}
\hline Sl.No & $\begin{array}{l}\text { Marital } \\
\text { Status }\end{array}$ & Group 'A' & \multicolumn{3}{l|}{ Group 'B' } & \multicolumn{2}{l|}{ Group 'C' } \\
\cline { 3 - 8 } & $\begin{array}{l}\text { No. of } \\
\text { Patients }\end{array}$ & Percentage & $\begin{array}{l}\text { No. of } \\
\text { Patients }\end{array}$ & Percentage & $\begin{array}{l}\text { No. } \\
\text { Patients }\end{array}$ & of \\
\hline 1 & Un married & 2 & $20 \%$ & 3 & $30 \%$ & 2 & $20 \%$ \\
\hline 2 & Married & 8 & $80 \%$ & 6 & $60 \%$ & 7 & $70 \%$ \\
\hline 3 & Widow & - & - & 1 & $10 \%$ & 1 & $10 \%$ \\
\hline Total & 10 & $100 \%$ & 10 & $100 \%$ & 10 & $100 \%$ \\
\hline
\end{tabular}

Table 6: Distribution of 30 patients of Kaphaja unmada according to Satisfaction in life events

\begin{tabular}{|l|l|l|l|l|l|l|l|}
\hline Sl.No & \multirow{2}{*}{ Stress events } & Group 'A' & Group 'B' & Group 'C' \\
\cline { 3 - 8 } & & Satisfaction & Un satisfaction & Satisfaction & $\begin{array}{l}\text { Un } \\
\text { satisfaction }\end{array}$ & Satisfaction & $\begin{array}{l}\text { Un } \\
\text { satisfaction }\end{array}$ \\
\hline 1 & Sexual life & 6 & 2 & 3 & 3 & - & 3 \\
\hline 2 & $\begin{array}{l}\text { Occupation } \\
\text { life }\end{array}$ & 1 & 9 & 5 & 5 & - & 4 \\
\hline
\end{tabular}


Table 7: Distribution of 30 patients of Kaphaja unmada according to disease chronicity

\begin{tabular}{|c|c|c|c|c|c|c|c|}
\hline \multirow[t]{2}{*}{ Sl.No } & \multirow{2}{*}{$\begin{array}{l}\text { Duration (in } \\
\text { years) }\end{array}$} & \multicolumn{2}{|l|}{ Group 'A' } & \multicolumn{2}{|l|}{ Group 'B' } & \multicolumn{2}{|l|}{ Group 'C' } \\
\hline & & $\begin{array}{ll}\text { No. } & \text { of } \\
\text { Patients }\end{array}$ & Percentage & $\begin{array}{ll}\text { No. } & \text { of } \\
\text { Patients }\end{array}$ & Percentage & $\begin{array}{ll}\text { No. } & \text { of } \\
\text { Patients } & \\
\end{array}$ & Percentage \\
\hline 1 & Up to $1 \mathrm{yrs}$ & 5 & $50 \%$ & 2 & $20 \%$ & 2 & $20 \%$ \\
\hline 2 & $1-3$ yrs & 3 & $30 \%$ & 2 & $20 \%$ & 1 & $10 \%$ \\
\hline 3 & $3-6$ & 1 & $10 \%$ & 3 & $30 \%$ & 3 & $30 \%$ \\
\hline 4 & $6-9$ & - & - & - & - & 1 & $10 \%$ \\
\hline 5 & $9-12$ & - & - & - & - & 1 & $10 \%$ \\
\hline 6 & $12-15$ & 1 & $10 \%$ & 2 & $20 \%$ & 3 & $30 \%$ \\
\hline Total & & 10 & $100 \%$ & 10 & $100 \%$ & 10 & $100 \%$ \\
\hline
\end{tabular}

Table 8: Distribution of 30 patients of Kaphaja unmada according to Stress

\begin{tabular}{|l|l|l|l|l|l|l|l|}
\hline Sl.No & Stress events & Group 'A' & \multicolumn{2}{l|}{ Group 'B' } & \multicolumn{2}{l|}{ Group 'C' } \\
\cline { 3 - 8 } & $\begin{array}{l}\text { No. of } \\
\text { Patients }\end{array}$ & Percentage & $\begin{array}{l}\text { No. } \\
\text { Patients }\end{array}$ & Percentage & $\begin{array}{l}\text { No. } \\
\text { Patients }\end{array}$ & Percentage \\
\hline 1 & $\begin{array}{l}\text { Family } \\
\text { problems }\end{array}$ & 8 & $80 \%$ & 6 & $60 \%$ & 5 & $50 \%$ \\
\hline 2 & $\begin{array}{l}\text { Relationship } \\
\text { with spouse }\end{array}$ & $2-6$ & $60 \%$ & 7 & $70 \%$ & 5 & $50 \%$ \\
\hline
\end{tabular}

Table 9: Distribution of 30 patients of Kaphaja unmada according to positive family history

\begin{tabular}{|l|l|l|l|l|l|l|l|}
\hline Sl.No & \multirow{2}{*}{ History of Family } & \multicolumn{2}{l|}{ Group 'A' } & \multicolumn{2}{l|}{ Group 'B' } & \multicolumn{2}{l|}{ Group 'C' } \\
\cline { 3 - 8 } & & $\begin{array}{l}\text { No. of } \\
\text { Patients }\end{array}$ & Percentage & $\begin{array}{l}\text { No. of } \\
\text { Patients }\end{array}$ & Percentage & $\begin{array}{l}\text { No. of } \\
\text { Patients }\end{array}$ & Percentage \\
\hline 1 & Psychiatric illness in family & 7 & $70 \%$ & 5 & $50 \%$ & 6 & $60 \%$ \\
\hline 2 & Alcohol or drug abuse in family & 3 & $30 \%$ & 7 & $70 \%$ & 6 & $60 \%$ \\
\hline 3 & Dearth in family & 3 & $30 \%$ & 2 & $20 \%$ & 4 & $40 \%$ \\
\hline 4 & Suicidal history in family & 3 & $30 \%$ & 2 & $20 \%$ & 4 & $40 \%$ \\
\hline
\end{tabular}

Table 10: Distribution of 30 patients of Kaphaja unmada according to Symptoms

\begin{tabular}{|c|c|c|c|c|c|c|}
\hline \multirow[t]{2}{*}{ Sl.No } & \multirow{2}{*}{$\begin{array}{l}\text { General } \\
\text { Symptoms }\end{array}$} & \multicolumn{3}{|c|}{ No. of Patients } & \multirow[t]{2}{*}{ Total } & \multirow[t]{2}{*}{ Percentage } \\
\hline & & Group 'A' & Group 'B' & Group 'C' & & \\
\hline 1 & Arochaks & 10 & 9 & 8 & 27 & $90 \%$ \\
\hline 2 & Chardi & 8 & 8 & 7 & 23 & 77 \\
\hline 3 & Alpaahara & 9 & 10 & 9 & 28 & 93 \\
\hline 4 & Alpachesta & 10 & 10 & 9 & 29 & 97 \\
\hline 5 & Alpavakya & 8 & 10 & 8 & 26 & 87 \\
\hline 6 & Alpamati & 9 & 10 & 9 & 28 & 93 \\
\hline 7 & Naripriyata & 5 & 2 & 3 & 10 & 33 \\
\hline 8 & Atinidrata & 6 & 5 & 6 & 17 & 57 \\
\hline 9 & Sadana & 9 & 10 & 9 & 28 & 93 \\
\hline 10 & Lalasrava & 9 & 7 & 6 & 22 & 73 \\
\hline 11 & Nakhadishauklyam & 8 & 7 & 7 & 22 & 73 \\
\hline 12 & Mukhashotha & 5 & 4 & 8 & 9 & 57 \\
\hline 13 & Ushnasevi & 6 & 6 & 7 & 19 & 63 \\
\hline 14 & Bibhatsya & 5 & 2 & 2 & 9 & 30 \\
\hline 15 & Shauchvidvesh & 8 & 6 & 8 & 22 & 73 \\
\hline 16 & Bhukebalam & 6 & 8 & 8 & 22 & 73 \\
\hline 17 & $\begin{array}{l}\text { Ratraubhrisham } \\
\text { bhavati }\end{array}$ & 6 & 5 & 5 & 17 & 57 \\
\hline
\end{tabular}


Anuradha et al

Journal of Drug Delivery \& Therapeutics; 2012, 2(5), 90-96

Table 11: Distribution of 30 patients of Kaphaja unmada according to Symptoms of Hamilton's Depression Rating Scale

\begin{tabular}{|c|c|c|c|c|c|c|}
\hline \multirow[t]{2}{*}{ Sl.No } & \multirow{2}{*}{ General Symptoms } & \multicolumn{3}{|c|}{ No. of Patients } & \multirow{2}{*}{ Total } & \multirow[t]{2}{*}{ Percentage } \\
\hline & & Group 'A' & Group 'B' & Group 'C' & & \\
\hline 1 & Depressed Mood & 10 & 10 & 10 & 30 & 100 \\
\hline 2 & Feeling of Guilt & 9 & 8 & 8 & 25 & 83 \\
\hline 3 & Suicidal tendency & 10 & 9 & 8 & 27 & 90 \\
\hline 4 & Early insomnia & 4 & 4 & 4 & 12 & 40 \\
\hline 5 & Insomnia middle & 1 & 2 & 3 & 6 & 20 \\
\hline 6 & Insomnia late & 3 & 2 & 4 & 9 & 30 \\
\hline 7 & Reduce work \& activity & 10 & 10 & 10 & 30 & 100 \\
\hline 8 & Retardations & 10 & 10 & 10 & 30 & 100 \\
\hline 9 & Agitation & 2 & 2 & 4 & 8 & 30 \\
\hline 10 & Anxiety & 8 & 7 & 8 & 23 & 77 \\
\hline 11 & Anxiety (somatic) & 6 & 5 & 8 & 19 & 63 \\
\hline 12 & Somatic Symptoms (GIT) & 6 & 5 & 7 & 18 & 60 \\
\hline 13 & Somatic Symptoms (General) & 2 & 4 & 6 & 12 & 40 \\
\hline 14 & Genital Symptoms & 7 & 5 & 5 & 17 & 57 \\
\hline 15 & Hypochondrias & 1 & 4 & 4 & 9 & 30 \\
\hline 16 & Loss of Weight & 10 & 6 & 5 & 21 & 70 \\
\hline 17 & Insight absent & 4 & 3 & 3 & 10 & 33 \\
\hline 18 & Diurnal variation & 6 & 6 & 8 & 20 & 67 \\
\hline 19 & Derealization & 1 & 2 & 1 & 4 & 13 \\
\hline 20 & Paranoid symptoms & 9 & 5 & 3 & 17 & 57 \\
\hline 21 & $\begin{array}{l}\text { Obsession and Compulsive } \\
\text { symptoms }\end{array}$ & 5 & 5 & 4 & 14 & 47 \\
\hline 22 & Helplessness & 10 & 8 & 9 & 27 & 90 \\
\hline 23 & Hopelessness & 10 & 10 & 10 & 30 & 100 \\
\hline 24 & Worthlessness & 10 & 10 & 10 & 30 & 100 \\
\hline
\end{tabular}

\section{RESULTS:}

Using score for kaphaja unmada rating scale \& Hamilton depression rating scale, assessment of the therapy was done. Mean scores before and after the intervention were noted and relief in percentage was calculated.

Table 12: Distribution of 30 patients of Kaphaja unmada according to Effect of Therapy on individual symptoms

\begin{tabular}{|c|c|c|c|c|c|c|c|c|}
\hline Symptoms & Group & B.T & A.T & $\begin{array}{l}\text { Mean } \\
\text { difference }\end{array}$ & Relief \% & St.Deviation & 't' & 'p' \\
\hline \multirow{3}{*}{ Arochaka } & A & 2.5 & 1.4 & 1.1 & 44 & 0.7379 & 4.714 & $<0.01$ \\
\hline & B & 2.0 & 1.0 & 1.0 & 50 & 0.6667 & 4.749 & $<0.01$ \\
\hline & $\mathrm{C}$ & 2.5 & 0.7 & 1.8 & 72 & 0.7888 & 7.216 & $<0.001$ \\
\hline \multirow[t]{3}{*}{ Chardi } & $\mathrm{A}$ & 1.9 & 0.7 & 1.2 & 63 & 1.0328 & 3.674 & $<0.05$ \\
\hline & B & 2.0 & 1.1 & 0.9 & 45 & 0.5676 & 5.104 & $<0.01$ \\
\hline & $\mathrm{C}$ & 1.6 & 0.4 & 1.2 & 75 & 0.8498 & 5.582 & $<0.001$ \\
\hline \multirow{3}{*}{ Alpaahara } & A & 2.7 & 1.1 & 1.6 & 59.25 & 0.6992 & 7.326 & $<0.001$ \\
\hline & $\mathrm{B}$ & 2.2 & 1.1 & 1.3 & 59.09 & 0.8498 & 5.582 & $<0.001$ \\
\hline & $\mathrm{C}$ & 1.8 & 0.5 & 1.3 & 72.22 & 0.8433 & 6.000 & $<0.001$ \\
\hline \multirow[t]{3}{*}{ Alpacheshta } & $\mathrm{A}$ & 2.6 & 1.4 & 1.2 & 46.15 & 0.4216 & 9.000 & $<0.001$ \\
\hline & $\mathrm{B}$ & 2.4 & 1.4 & 1.0 & 41.66 & 0.4714 & 6.708 & $<0.001$ \\
\hline & $\mathrm{C}$ & 2.6 & 0.5 & 2.1 & 80.76 & 0.3162 & 21.000 & $<0.001$ \\
\hline \multirow[t]{3}{*}{ Alpavakyta } & A & 2.7 & 1.6 & 1.1 & 40.74 & 0.5676 & 6.128 & $<0.001$ \\
\hline & $\mathrm{B}$ & 2.1 & 1.1 & 1.0 & 47.61 & 0.6667 & 4.473 & $<0.01$ \\
\hline & $\mathrm{C}$ & 2.2 & 0.4 & 1.8 & 81.81 & 1.0593 & 5.075 & $<0.01$ \\
\hline \multirow[t]{3}{*}{ Alpamati } & $\mathrm{A}$ & 2.7 & 1.6 & 1.1 & 40.74 & 0.3162 & 11.000 & $<0.001$ \\
\hline & B & 2.3 & 1.1 & 1.2 & 52.17 & 0.6325 & 6.000 & $<0.001$ \\
\hline & $\mathrm{C}$ & 2.2 & 0.6 & 1.6 & 72.72 & 0.8433 & 6.000 & $<0.001$ \\
\hline \multirow[t]{3}{*}{ Naripriyata } & A & 0.5 & 0.3 & 0.2 & 40.00 & 0.4216 & 1.500 & $<0.05$ \\
\hline & B & 0.8 & 0.5 & 0.3 & 37.50 & 0.4830 & 1.964 & $<0.01$ \\
\hline & $\mathrm{C}$ & 1.3 & 0.3 & 1.0 & 76.92 & 1.1547 & 1.500 & $<0.05$ \\
\hline \multirow{3}{*}{$\begin{array}{l}\text { Vivikta } \\
\text { Priyata }\end{array}$} & A & 2.8 & 1.7 & 1.1 & 39.28 & 0.3162 & 11.000 & $<0.001$ \\
\hline & B & 2.4 & 1.3 & 1.1 & 45.83 & 0.5676 & 6.128 & $<0.001$ \\
\hline & C & 2.3 & 0.5 & 1.8 & 78.26 & 1.1353 & 5.014 & $<0.01$ \\
\hline
\end{tabular}




\begin{tabular}{|c|c|c|c|c|c|c|c|c|}
\hline \multicolumn{9}{|c|}{2 Continue.......... } \\
\hline Symptoms & Group & B.T & A.T & $\begin{array}{l}\text { Mean } \\
\text { difference }\end{array}$ & Relief \% & St.Deviation & ' $t$ ' & 'p' \\
\hline \multirow[t]{3}{*}{ Atinidrata } & $\mathrm{A}$ & 1.5 & 1.0 & 0.5 & 33.33 & 0.5270 & 3.000 & $<0.05$ \\
\hline & $\mathrm{B}$ & 1.7 & 1.0 & 0.7 & 41.17 & 0.6749 & 3.280 & $<0.05$ \\
\hline & $\mathrm{C}$ & 1.6 & 0.4 & 1.2 & 75 & 1.0328 & 3.674 & $<0.05$ \\
\hline \multirow[t]{3}{*}{ Sodana } & A & 2.8 & 1.5 & 1.3 & 46.42 & 0.4830 & 8.510 & $<0.001$ \\
\hline & B & 2.5 & 1.5 & 1.0 & 40 & 0.4714 & 6.708 & $<0.001$ \\
\hline & $\mathrm{C}$ & 2.3 & 0.5 & 1.8 & 78.26 & 0.9189 & 6.194 & $<0.001$ \\
\hline \multirow[t]{3}{*}{ Lalasrava } & $\mathrm{A}$ & 2.0 & 1.3 & 0.7 & 35 & 0.4830 & 4.583 & $<0.01$ \\
\hline & B & 1.7 & 0.9 & 0.8 & 47.05 & 0.7888 & 3.207 & $<0.05$ \\
\hline & $\mathrm{C}$ & 2.3 & 0.7 & 1.6 & 69.56 & 0.6992 & 7.236 & $<0.001$ \\
\hline \multirow{3}{*}{$\begin{array}{l}\text { Nakhadisau } \\
\text { klyam }\end{array}$} & A & 1.9 & 0.6 & 0.7 & 36.84 & 0.4830 & 4.583 & $<0.01$ \\
\hline & B & 1.9 & 1.1 & 0.8 & 42.10 & 0.6325 & 4.000 & $<0.05$ \\
\hline & $\mathrm{C}$ & 2.2 & 0.5 & 1.7 & 77.27 & 1.0593 & 5.075 & $<0.01$ \\
\hline \multirow[t]{3}{*}{ Ushnasevi } & $\mathrm{A}$ & 1.5 & 0.8 & 0.7 & 46.66 & 0.6744 & 3.280 & $<0.05$ \\
\hline & $\mathrm{B}$ & 1.9 & 1.0 & 0.9 & 47.36 & 0.8756 & 3.250 & $<0.05$ \\
\hline & $\mathrm{C}$ & 1.6 & 0.4 & 1.2 & 75 & 1.2293 & 3.087 & $<0.05$ \\
\hline \multirow{3}{*}{$\begin{array}{l}\text { Mukhashot } \\
\text { ha }\end{array}$} & $\mathrm{A}$ & 1.0 & 0.6 & 0.4 & 40 & 1.1972 & 2.905 & $<0.05$ \\
\hline & B & 2.2 & 1.2 & 0.2 & 45.45 & 0.5164 & 2.449 & $<0.01$ \\
\hline & $\mathrm{C}$ & 1.3 & 0.2 & 1.1 & 84.61 & 1.1972 & 2.905 & $<0.05$ \\
\hline \multirow[t]{3}{*}{ Bhibhatsya } & A & 0.5 & 0.4 & 1.0 & 20.92 & 0.5676 & 0.557 & $<0.05$ \\
\hline & B & 0.6 & 0.4 & 0.1 & 33.33 & 0.4214 & 1.500 & $<0.05$ \\
\hline & $\mathrm{C}$ & 1.3 & 0.3 & 1.0 & 76.92 & 1.1547 & 2.739 & $<0.05$ \\
\hline \multirow{3}{*}{$\begin{array}{l}\text { Sanchvidva } \\
\text { m }\end{array}$} & $\mathrm{A}$ & 1.7 & 1.1 & 0.6 & 35.29 & 0.5164 & 3.674 & $<0.01$ \\
\hline & B & 2.0 & 1.0 & 1.0 & 50 & 0.6667 & 4.743 & $<0.01$ \\
\hline & $\mathrm{C}$ & 2.1 & 0.5 & 1.6 & 76.19 & 0.8433 & 6.000 & $<0.001$ \\
\hline \multirow{3}{*}{$\begin{array}{l}\text { Bhuktebala } \\
\text { m }\end{array}$} & A & 2.3 & 1.4 & 0.9 & 39.13 & 0.5676 & 5.014 & $<0.01$ \\
\hline & B & 2.1 & 1.1 & 1.0 & 47.61 & 0.6667 & 4.743 & $<0.01$ \\
\hline & $\mathrm{C}$ & 1.7 & 0.4 & 1.3 & 76.47 & 1.1595 & 3.545 & $<0.01$ \\
\hline \multirow{3}{*}{$\begin{array}{l}\text { Ratraubjris } \\
\text { ham bhavati }\end{array}$} & $\mathrm{A}$ & 1.4 & 0.9 & 0.3 & 21.42 & 0.7071 & 2.236 & $<0.05$ \\
\hline & B & 1.7 & 0.5 & 1.2 & 70.58 & 0.9487 & 2.333 & $<0.05$ \\
\hline & $\mathrm{C}$ & 1.6 & 0.5 & 1.1 & 68.75 & 1.1005 & 3.161 & $<0.05$ \\
\hline
\end{tabular}

Table 13: Effect of Therapy on signs \& symptoms of according to HDRS

\begin{tabular}{|c|c|c|c|c|c|c|c|c|}
\hline Symptoms & Group & B.T & A.T & $\begin{array}{l}\text { Mean } \\
\text { difference }\end{array}$ & $\begin{array}{l}\text { Relief } \\
\%\end{array}$ & St.Deviation & 't' & 'p' \\
\hline \multirow[t]{3}{*}{ Depressed mood } & $\mathrm{A}$ & 3.4 & 1.9 & 2.5 & 73.52 & 0.7071 & 6.708 & $<0.001$ \\
\hline & B & 3.2 & 2.0 & 1.5 & 37.5 & 0.4216 & 9.000 & $<0.001$ \\
\hline & $\mathrm{C}$ & 3.7 & 0.6 & 3.1 & 83.78 & 0.5676 & 17.270 & $<0.001$ \\
\hline \multirow[t]{3}{*}{ Feeling of guilt } & $\mathrm{A}$ & 2.9 & 1.8 & 1.1 & 37.93 & 0.8756 & \begin{tabular}{|l|}
3.973 \\
\end{tabular} & $<0.01$ \\
\hline & B & 2.9 & 1.6 & 1.3 & 44.82 & 0.7888 & 4.811 & $<0.01$ \\
\hline & $\mathrm{C}$ & 3.1 & 0.5 & 2.6 & 83.87 & 1.1738 & 7.005 & $<0.001$ \\
\hline \multirow{3}{*}{ Suicidal thinking } & $\bar{A}$ & 3.2 & 1.4 & 1.8 & 56.25 & 0.7888 & \begin{tabular}{|l|}
7.216 \\
\end{tabular} & $<0.001$ \\
\hline & B & 2.5 & 1.2 & 1.3 & 52 & 0.8233 & 4.993 & $<0.01$ \\
\hline & $\mathrm{C}$ & 3.2 & 0.8 & 2.4 & 75 & 0.8433 & 9.00 & $<0.001$ \\
\hline \multirow[t]{3}{*}{ Insomnia early } & $\mathrm{A}$ & 0.5 & 0.2 & 0.3 & 60 & 0.6749 & 1.406 & $<0.05$ \\
\hline & B & 0.5 & \begin{tabular}{|l|}
0.3 \\
\end{tabular} & 0.2 & 40 & 0.6749 & $\begin{array}{ll}1.406 \\
\end{array}$ & $>0.05$ \\
\hline & $\bar{C}$ & 0.8 & 0.2 & 0.6 & 75 & 0.564 & 3.674 & $<0.01$ \\
\hline \multirow{3}{*}{ Insomnia middle } & $\mathrm{A}$ & 0.1 & 0.0 & 0.1 & 100 & 0.3162 & 1.000 & $>0.05$ \\
\hline & B & 0.3 & 0 & 0.3 & 100 & 0.6749 & 1.406 & $>0.05$ \\
\hline & $\mathrm{C}$ & 0.5 & 0.1 & 0.4 & 80 & 0.6992 & 1.809 & $>0.05$ \\
\hline \multirow{3}{*}{ Insomnia late } & $\mathrm{A}$ & 0.3 & $\begin{array}{ll}0.1 \\
\end{array}$ & 0.2 & 66.66 & 0.4216 & 3.674 & $>0.05$ \\
\hline & $\mathrm{B}$ & 0.3 & 0.2 & 0.3 & 60 & 0.6749 & 3.545 & $>0.05$ \\
\hline & $\mathrm{C}$ & 0.5 & \begin{tabular}{|l|}
0.1 \\
\end{tabular} & 0.2 & 66.66 & 0.4216 & 8.135 & $>0.05$ \\
\hline \multirow{3}{*}{$\begin{array}{l}\text { Diminished activity } \\
\text { \& work }\end{array}$} & $\bar{A}$ & 2.6 & 1.4 & 1.2 & 46.15 & 1.0328 & 3.674 & $<0.01$ \\
\hline & $\mathrm{B}$ & 2.9 & 1.6 & 1.3 & 44.82 & 1.1595 & 3.545 & $<0.01$ \\
\hline & $\mathrm{C}$ & 3.1 & 0.6 & 2.5 & 80.64 & 0.9718 & 8.135 & $<0.001$ \\
\hline \multirow[t]{3}{*}{ Retardation } & A & 3.5 & 1.4 & 2.1 & 60.00 & 0.7379 & 9.000 & $<0.001$ \\
\hline & B & 3.6 & 1.6 & 2.0 & 55.55 & 0.6667 & 9.487 & $<0.001$ \\
\hline & $\mathrm{C}$ & 3.5 & 1.2 & 2.3 & 65.71 & 0.6749 & 10.776 & $<0.001$ \\
\hline
\end{tabular}




\begin{tabular}{|c|c|c|c|c|c|c|c|c|}
\hline \multicolumn{9}{|c|}{ Table 13 Continue. } \\
\hline Symptoms & Group & B.I & & $\begin{array}{l}\text { Mean } \\
\text { difference }\end{array}$ & $\begin{array}{l}\text { Kellet } \\
\%\end{array}$ & St.Deviation & $\mathbf{t}^{\prime}$ & $\mathbf{p}$ \\
\hline \multirow[t]{3}{*}{ Agitation } & A & 0.6 & 0.4 & 0.2 & 33.33 & 0.6235 & 1.000 & $>0.05$ \\
\hline & $\mathrm{B}$ & 1.0 & 0.5 & 0.5 & 50 & 0.7071 & 2.236 & $>0.05$ \\
\hline & $\mathrm{C}$ & 0.5 & 0.3 & 0.2 & 40 & 0.4216 & 1.500 & $>0.05$ \\
\hline \multirow[t]{3}{*}{ Anxiety(Psychic) } & A & 2.3 & 1.1 & 1.25 & 52.17 & 1.0328 & 3.674 & $<0.01$ \\
\hline & B & 2.3 & 1.6 & 0.7 & 30.43 & 0.6749 & 3.280 & $<0.05$ \\
\hline & $\mathrm{C}$ & 2.5 & 1.2 & 1.3 & 52 & 0.9847 & 4.333 & $<0.01$ \\
\hline \multirow[t]{3}{*}{ Anxiety somatic } & $\bar{A}$ & 1.2 & \begin{tabular}{|l|l|}
0.7 \\
\end{tabular} & 0.5 & 41.66 & 0.8498 & 1.861 & $<0.05$ \\
\hline & $\mathrm{B}$ & 2.5 & 1.2 & 1.3 & 52 & 0.0593 & 3.881 & $>0.05$ \\
\hline & $\mathrm{C}$ & 1.7 & 0.5 & 1.2 & 70.58 & 1.5492 & 2.449 & $<0.01$ \\
\hline \multirow{3}{*}{$\begin{array}{l}\text { Somatic } \\
\text { Symptoms } \\
\text { G.I.T }\end{array}$} & A & 1.2 & 0.6 & 0.6 & 50 & 0.6992 & 2.174 & $<0.05$ \\
\hline & B & 1.9 & 1.3 & 0.6 & 31.57 & 0.6992 & 2.174 & $>0.05$ \\
\hline & $\mathrm{C}$ & 1.4 & 0.2 & 1.2 & 85.71 & 1.229 & 3.087 & $<0.05$ \\
\hline \multirow{3}{*}{$\begin{array}{l}\text { Somatic } \\
\text { Symptoms } \\
\text { General }\end{array}$} & $\overline{\mathrm{A}}$ & 1.3 & 0.6 & 0.6 & 53.84 & 0.9487 & 2.333 & $<0.05$ \\
\hline & B & 1.9 & 1.0 & 1.0 & 47.36 & 0.9944 & 2.862 & $<0.05$ \\
\hline & $\mathrm{C}$ & 0.5 & 0.3 & 0.3 & 40 & 0.4216 & 1.500 & $>0.05$ \\
\hline \multirow{3}{*}{$\begin{array}{l}\text { Genital } \\
\text { Symptoms }\end{array}$} & A & 1.3 & 0.6 & 0.7 & 53.58 & 0.8233 & 2.689 & $<0.05$ \\
\hline & B & 1.9 & 1.0 & 0.9 & 47.36 & 0.8233 & 2.689 & $<0.05$ \\
\hline & $\mathrm{C}$ & 0.5 & 0.3 & 0.2 & 40 & 0.7888 & 3.207 & $<0.05$ \\
\hline \multirow[t]{3}{*}{ Hypochondria } & A & 0.9 & 0.5 & 0.7 & 44.44 & 0.5164 & 2.449 & $<0.05$ \\
\hline & B & 0.8 & 0.3 & 0.9 & 62.5 & 0.7071 & 2.236 & $>0.05$ \\
\hline & $\mathrm{C}$ & 0.2 & 0.3 & 0.2 & 0 & 0.4216 & 1.500 & $>0.05$ \\
\hline \multirow{3}{*}{ Loss of weight } & $\bar{A}$ & 1.1 & 0.2 & 0.4 & 63.63 & 0.9487 & 2.333 & $>0.05$ \\
\hline & $\mathrm{B}$ & 0.8 & 0.4 & 0.5 & 60 & 0.4830 & 1.964 & $<0.05$ \\
\hline & $\mathrm{C}$ & 1.9 & 0.5 & 0 & 52.63 & 0.8165 & 3.873 & $>0.05$ \\
\hline \multirow[t]{3}{*}{ Insight absent } & A & 0.3 & 0.9 & 0.7 & 66.66 & 0.4216 & 1.500 & $<0.01$ \\
\hline & B & 0.3 & 0.1 & 0.3 & 66.66 & 0.4216 & 1.500 & $>0.05$ \\
\hline & $\mathrm{C}$ & 0.7 & \begin{tabular}{|l|l|}
0.1 \\
\end{tabular} & 1.0 & 71.42 & 0.5270 & 3.000 & $>0.05$ \\
\hline \multirow[t]{3}{*}{ Diurnal } & A & 1.1 & 0.2 & 0.2 & 54.54 & 0.6992 & 2.714 & $<0.05$ \\
\hline & $\mathrm{B}$ & 1.9 & 0.5 & 0.2 & 15.78 & 0.8233 & 1.152 & $<0.05$ \\
\hline & $\mathrm{C}$ & 1.4 & 1.6 & 0.5 & 64.28 & 0.9944 & 2.862 & $>0.05$ \\
\hline \multirow{3}{*}{$\begin{array}{l}\text { Depersonalization } \\
\text { \&Derealisation }\end{array}$} & A & 0.3 & 0.5 & 0.6 & 66.66 & 0.4216 & 1.500 & $<0.05$ \\
\hline & $\mathrm{B}$ & 0.2 & 0.3 & 0.3 & 100 & 0.6325 & 1.000 & $>0.05$ \\
\hline & $\mathrm{C}$ & 0.2 & 0.1 & 0.9 & 100 & 0.6325 & 1.000 & $>0.05$ \\
\hline \multirow{3}{*}{$\begin{array}{l}\text { Paranoid } \\
\text { Symptoms }\end{array}$} & $\bar{A}$ & 0.6 & 0 & 0.2 & 50 & 0.4830 & 1.964 & $>0.05$ \\
\hline & B & 1.1 & 0 & 0.2 & 45.45 & 0.7071 & 2.236 & $>0.05$ \\
\hline & $\mathrm{C}$ & 1.8 & 0.3 & 0.2 & 64.28 & 0.5676 & 5.014 & $<0.01$ \\
\hline \multirow{3}{*}{$\begin{array}{l}\text { Obsession } \\
\text { \&compulsive } \\
\text { systems }\end{array}$} & A & 1.1 & 0.6 & 0.5 & 45.45 & 0.7071 & 2.236 & $>0.05$ \\
\hline & B & 1.4 & 0.7 & 0.7 & 50 & 1.2517 & 1.769 & $>0.05$ \\
\hline & $\mathrm{C}$ & 1.4 & 0.5 & 0.9 & 62.28 & 1.1005 & 2.586 & $<0.05$ \\
\hline \multirow{3}{*}{ Helplessness } & A & 2.3 & 1.4 & 0.9 & 39.13 & 0.7379 & 3.857 & $<0.01$ \\
\hline & B & 2.0 & \begin{tabular}{|l|}
0.9 \\
\end{tabular} & 1.1 & 55 & 0.7379 & 4.714 & $<0.01$ \\
\hline & $\mathrm{C}$ & 2.8 & \begin{tabular}{|l|}
1.2 \\
\end{tabular} & 1.6 & 57.14 & 0.6992 & 7.236 & $<0.001$ \\
\hline \multirow[t]{3}{*}{ Hopelessness } & A & 3.3 & 2.3 & 1.0 & 30.30 & 0.9428 & 3.354 & $<0.01$ \\
\hline & B & 3.4 & \begin{tabular}{|l|}
1.7 \\
\end{tabular} & 1.7 & 50 & 0.8233 & 6.530 & $<0.001$ \\
\hline & $\mathrm{C}$ & 3.6 & 1.3 & 2.6 & 72.22 & 0.6749 & 10.776 & $<0.001$ \\
\hline \multirow[t]{3}{*}{ Worth less ness } & A & 3.2 & \begin{tabular}{|l|}
1.9 \\
\end{tabular} & 1.3 & 40.62 & 0.9487 & 4.333 & $<0.001$ \\
\hline & B & 3.1 & \begin{tabular}{|l|}
1.2 \\
\end{tabular} & 1.9 & 61.29 & 0.8756 & 6.862 & $<0.01$ \\
\hline & $\mathrm{C}$ & 2.9 & 1.3 & 1.6 & 55.17 & 0.5164 & 9.778 & $<0.001$ \\
\hline
\end{tabular}

The change occurred in all groups was statistically highly significant.

Table 14: Comparison of effects of treatment on KU Scale between the groups (A, B\&C)

\begin{tabular}{|l|l|l|l|l|l|l|l|}
\hline Group & B.T & A.T & $\begin{array}{l}\text { Mean } \\
\text { difference }\end{array}$ & Relief \% & St.Deviation & 't' & 'p' \\
\hline A & 34.7 & 19.8 & 14.9 & 42.93 & 2.1318 & 22.103 & $>0.001$ \\
\hline B & 34.4 & 18.2 & 16.2 & 47.09 & 2.290 & 23.275 & $>0.001$ \\
\hline C & 35.1 & 8.3 & 26.8 & 76.35 & 4.3665 & 19.409 & $<0.001$ \\
\hline
\end{tabular}

The score of signs and symptoms was changed from 35.1 to 8.3 in 45 days and relief was $76.35 \%$ in the ' $\mathrm{C}$ ' group (10) $(\mathrm{P}<.001)$. The change was significant. While in group ' $\mathrm{B}$ ' mean change was from 34.4 to 18.2 within 45 days and relief was $47.09 \%<\mathrm{P}<0.001$. The change was significant. 
Table 15: Comparison of effects of treatment on HDR Scale between the groups

\begin{tabular}{|l|l|l|l|l|l|l|l|}
\hline Group & B.T & A.T & Mean difference & Relief \% & St.Deviation & 't' & 'p' \\
\hline A & 38.3 & 20.3 & 18.0 & 46.99 & 5.9815 & 10.045 & $>0.001$ \\
\hline B & 42.2 & 22.0 & 20.2 & 47.86 & 3.7653 & 3.7653 & $>0.001$ \\
\hline C & 47.8 & 16.2 & 31.6 & 66.10 & 8.4485 & 11.828 & $<0.001$ \\
\hline
\end{tabular}

The change occurred in group ' $\mathrm{C}$ ' is highly significant and the change occurred in both 'A\&B' groups is statistically significant.

Table 16: grading for the results of Kaphaja unmade

\begin{tabular}{|l|l|l|l|l|l|l|l|}
\hline Sl.No & Grade & Group 'A' & Group 'B' & \multicolumn{2}{l|}{ Group 'C' } \\
\cline { 3 - 8 } & & No. of Patients & $\mathbf{\%}$ & No. of Patients & \% & No. of Patients & \% \\
\hline 1 & Cured & 0 & 0 & 0 & 0 & 0 & 0 \\
\hline 2 & Marked improvement & 0 & 0 & 0 & 0 & 8 & $80 \%$ \\
\hline 3 & Moderate improvement & 3 & $30 \%$ & 1 & $10 \%$ & 2 & $20 \%$ \\
\hline 4 & Mild improvement & 7 & $70 \%$ & 9 & $90 \%$ & 0 & 0 \\
\hline 5 & Unchanged & 0 & 0 & 0 & 0 & 0 & 0 \\
\hline 6 & Worsened & 0 & 0 & 0 & 0 & 0 & 0 \\
\hline & Total: & 10 & 100 & 10 & 100 & 10 & 100 \\
\hline
\end{tabular}

Table 17: grading for the results of Hamilton's rating scale for depression

\begin{tabular}{|l|l|l|l|l|l|l|l|}
\hline Sl.No & Grade & Group 'A' & Group 'B' & Group 'C' \\
\cline { 3 - 8 } & & No. of Patients & $\mathbf{\%}$ & No. of Patients & $\mathbf{\%}$ & No. of Patients & \% \\
\hline 1 & Cured & 0 & 0 & 0 & 0 & 0 & 0 \\
\hline 2 & Marked improvement & 0 & 0 & 0 & 0 & 1 & $10 \%$ \\
\hline 3 & Same & 3 & $30 \%$ & 2 & $20 \%$ & 9 & $90 \%$ \\
\hline 4 & As & 7 & $70 \%$ & 8 & $80 \%$ & 0 & 0 \\
\hline 5 & Above & 0 & 0 & 0 & 0 & 0 & 0 \\
\hline 6 & Table & 0 & 0 & 0 & 0 & 0 & 0 \\
\hline & Total: & 10 & 100 & 10 & 100 & 10 & 100 \\
\hline
\end{tabular}

In group 'C' 1 patient got (10\%) marked improvement, 9 patients $(90 \%)$ got moderate improvement. in group ' $A$ ' 3 pts $(30 \%)$ got moderate improvement, 7patients $(70 \%)$ got mild improvement. In group 'B' 2 patients (20\%) got moderate improvement and 8 patients $(80 \%)$ got mild improvement.

\section{DISCUSSION:}

The main objective of this research is to verify the truth in our ancient concepts and to evolve practical ways of treatment relevant to the present era. Depression is emerging as a dreadful problem both as a tough disease to deal with and as a challenge for growth and development worldwide. Out of 23 symptoms of depressive disorders 17 of them are common to Kaphonmada (74\%).

In modern parlance Ghrita and Brain appear to be predominantly lipid in nature. Ghrita increases Bala (strength), Agni( appetite ) Medha ( intellect )and concentration capacity. All the ingredients of Hingwadi gritham are vata-kapha hara \& so it is expected to be very effective in Kaphaja unmada. According to all Ayurvedic acharyas Nasa is the portal of shiras (Head). It does not mean any anatomical channel that connects directly to the brain but it might be connected pharmacodynamically through blood vessels (or) through nervous systems (olfactory nerves etc). So Nasa is considered for sending the medicine to the brain.

\section{CONCLUSION:}

The definition of unmada provided by Acharya Charaka can be taken as general guideline to understand all mental disorders in terms of its Psychopathology. The symptoms of kaphaja unmada \& depression are approximately same. Highly significant results were observed in patients who were treated with both Nasya \& Pana (Nasal \& Oral) therapy with Hingwadi grutham. No objective parameters were used. The short period of study limits number of observations. To overcome the stress \& strain of modern life one need to follow the measures mentioned by Ayurvedic Acharyas aimed at bestowing relaxation \& mental balance, which are basic prerequisites of a sound mind. These measures are relevant even today.

ACKNOWLEDGMENT:I am very thankful to my children Alekhya \& Ajay who helped me in computing the research work.

\section{REFERENCES:}

1) Internet, google,Medical (Ayu)

2) Atharvaved 10:12:13

3) Agnivesa - Charaka Samhita revised by charaka \& Dhridabaka $5^{\text {th }}$ edition - Chowkamba Sanskrit Samsthana Varanasi-2001

4) Sabdasthama mahanidhi.

5) Astanga Hridaya with English translation translated by K.R.Srikanta Murthy, Krishnadas academy, Varanasi, $3^{\text {rd }}$ edition (2001).

6) Atharvaveda with padartha and Sayana, S. Commentary Vishwabandu Sri.Krishna Shastry, Venkateshwara printing press - Mumbai (19. 7)Bhavaprakasha of Bhavamisra with English translation Translated by Prof.K.R.Srikantha Murthy, Krishnadas Academy, Varanasi $1^{\text {st }}$ edition . 8)Charaka samhita with chakrapani commentry by Ganga sahey pande choukamba publica

9)Desai R.R, Nidana Chikista Hastamalak, Shree Baidyanath Ayurveda Bhavan Pvt.Ltd, Calcutta $6^{\text {th }}$ edition).

10)Nadkarni.KM.Indian Materia Medica, popular prakashan Pvt.Ltd. Mumbai, Second reprint of $3^{\text {rd }}$ revised edition (1982).

11)P.V.Sharma, History of Medicine in India, Indian National Science Academy N.Delhi (1992) $1^{\text {st }}$. 